\title{
On the sinterability of crystallizing glass powders
}

\author{
Miguel Oscar Prado ${ }^{\mathrm{a}, *}$, Marcio Luis Ferreira Nascimento ${ }^{\mathrm{b}, *}$, Edgar Dutra Zanotto ${ }^{\mathrm{b}, *}$ \\ ${ }^{a}$ Consejo Nacional de Investigaciones Científicas y Técnicas (CONICET), Comisión Nacional de Energía Atómica, Centro Atómico Bariloche, Av. Bustillo, km 9.5, \\ San Carlos de Bariloche, Argentina \\ ${ }^{\mathrm{b}}$ Laboratório de Materiais Vítreos, Universidade Federal de São Carlos, Av. Washington Luiz, km 325, 13.565-905, São Carlos, SP, Brazil ${ }^{1}$
}

\section{A R T I C L E I N F O}

\section{Article history:}

Received 15 August 2007

Received in revised form 19 May 2008

Available online 17 July 2008

\section{PACS:}

61.43.Fs

$66.20 .+\mathrm{d}$

81.20.Ev

81.40.Ef

Keywords:

Crystallization

Crystal growth

Diffusion and transport

Transport properties - liquids

Oxide glasses

Sintering

Viscosity

\begin{abstract}
A B S T R A C T
We define an adimensional parameter, $S$, that is capable of predicting if a glass powder compact can be fully densified by viscous flow sintering or if concurrent surface crystallization will hinder densification. The proposed sinterability parameter is $S(T)=\gamma /\left\lfloor\sqrt{N_{S}} \cdot U(T) \cdot \eta(T) \cdot r\right\rfloor$, where $\gamma$ is the glass-vapor surface energy, $N_{S}$ the density of nucleation sites on the glass surface, $U(T)$ the crystal growth rate, $\eta(T)$ the viscosity, and $r$ the average particle radius. For high temperatures, $T \geqslant 0.85 T_{\mathrm{m}}$, where $T_{\mathrm{m}}$ is the melting point of the crystal phase, an approximate expression can be used:

$S_{h T}(T)=2 \pi \cdot \gamma \cdot N_{\mathrm{A}} \cdot T_{\mathrm{m}}^{2} \cdot \sqrt[3]{V_{\mathrm{m}}^{2}} /\left[10 \sqrt{N_{\mathrm{S}}} \cdot r \cdot \Delta H_{\mathrm{m}} \cdot \Delta T^{2}\right]$,

where $V_{\mathrm{m}}$ is the molar volume, $N_{\mathrm{A}}$ is Avogadro's number, $\Delta H_{\mathrm{m}}$ is the melting enthalpy of the crystal phase, and $\Delta T=T_{\mathrm{m}}-T$ is the undercooling. This expression avoids the (time consuming) measurement of $U(T)$ and $\eta(T)$. Predictions can be made by $S$ or $S_{h T}$ thus avoiding the need of any sintering experiment. For a given glass-forming composition the physical properties are fixed, but higher temperatures and smaller particle sizes increase $S$ and privilege sintering over surface crystallization. We demonstrate that the condition to successfully densify any glass powder at a given temperature is $S>50$. This new parameter is a very useful aid for the development of sintered glasses and glass-ceramics.
\end{abstract}

(c) 2008 Elsevier B.V. All rights reserved.

\section{Introduction}

Glass sintering is an alternative technique to produce dense or porous glass articles or, when surface crystallization occurs one can also develop sintered glass-ceramics. Once a glass powder compact is heated above the glass transition temperature, $T_{\mathrm{g}}$, a race between sintering and crystallization begins. The powder's surface area and its associated surface energy tend to decrease through sintering, but a concurrent process, predominant surface crystallization in most glasses (or internal crystallization in a few glasses) also takes place to decrease the overall free energy of the glass transforming it into a polycrystalline material. Therefore, there are two simultaneous pathways for the system's free energy decrease, each one with its own kinetics: viscous flow sintering and crystallization.

Despite the extensive literature on glass sintering only a few papers analyzed the competition between sintering and surface crys-

\footnotetext{
* Corresponding authors. Tel.: +55 16 33518556; fax: +55 1633615404 .

E-mail addresses: pradom@cab.cnea.gov.ar (M.O. Prado), pmlfn@iris.ufscar.br (M.L.F. Nascimento), dedz@power.ufscar.br (E.D. Zanotto).

${ }^{1}$ http://www.lamav.ufscar.br.
}

tallization on a quantitative basis (e.g. Ref. [1]), i.e., by evaluating the influence of the glass surface energy, $\gamma$, viscosity, $\eta(T)$, particle size, $r$, surface density of nucleation sites, $N_{S}$, and crystal growth rate, $U(T)$, on the kinetics of these processes. Among all these properties only the viscosity and crystal growth rates strongly depend on temperature. In previous papers [2-6] we developed a model (the Clusters model) to describe the sintering kinetics of glass powder compacts undergoing or not concurrent surface crystallization. Besides crystallization, we considered a series of other complicating factors, such as irregular particle packing, poor surface quality of the glass grains (crystalline foreign inclusions), several simultaneously crystallizing phases, compositional shifts caused by crystallization, degassing, and glasses with embedded ceramic fibers.

As regards to non-isothermal sintering with concurrent crystallization, Müller [7] found, for instance, that to fully densify cordierite glass particles of about $1 \mu \mathrm{m}$, heating rates equal to or higher than $12 \mathrm{~K} / \mathrm{min}$ were necessary. This means that, at this heating rate, when $1 \mu \mathrm{m}$ particles reached the chosen sintering temperature, their surfaces had not or had only partially crystallized. Surface crystallization hindered sintering for lower heating rates. Prado et al. [6,8] confirmed Müller's results on the influence of the heating rate on the final density of any glass compact. 
For isothermal processes, Prado et al. [3,4] performed a detailed study of the kinetic competition between sintering and surface crystallization with a soda-lime-silica glass. Such studies demonstrated that depending on the intrinsic physicochemical parameters of the glass, such as viscosity, surface energy and crystal growth rate, as well as on experimental conditions (such as particle size, density of nucleation sites, time and temperature of heat treatment) some glass compacts may crystallize before full densification, or may fully sinter before crystallization begins; or yet some intermediate state between these two extreme behaviors may occur. In summary, their studies demonstrated that high temperatures, $T$, and high values of glass-vapor surface energy, $\gamma$, and low values of viscosity, $\eta(T)$, density of surface nucleation sites, $N_{\mathrm{S}}$, crystal growth rate, $U(T)$, and average particle size, $r$, favor densification over crystallization. These physicochemical properties depend exclusively on the glass composition, while $N_{\mathrm{S}}, r$ and $T$ are process variables that can be controlled to favor densification.

In this paper, we analyze the sinterability of crystallizing glass powders to establish the ability with which they will sinter when heated. We propose a parameter that can gauge whether or not a given glass powder compact can be densified (before any sintering experiment is carried out). The proposed sinterability parameter was tested and proved to establish the necessary combination of physical properties and processing conditions (time and temperature) for a crystallizing glass powder to achieve a high degree of densification.

\section{Theory}

To discuss the sinterability parameter, $S$, first of all it is necessary to briefly discuss the classical crystal growth mechanisms controlling surface crystallization kinetics. We then present a brief explanation about viscous flow sintering with concurrent crystallization and, finally, define a complete expression and a simplified form for $S$.

\subsection{Crystal growth mechanisms}

Two phenomenological models are frequently used to describe crystal growth kinetics controlled by atomic or molecular rearrangements at the crystal-liquid interface: normal $[9,10]$ or screw dislocation growth [11]. Growth controlled by $2 D$ surface nucleation is less frequent. According to Jackson's treatment of the interface, materials with small entropy of fusion, such as silica $[9,10]$ $\left(\Delta S_{\mathrm{m}}=0.46 R\right)$ or larger, as diopside [11] $\left(\Delta S_{\mathrm{m}}=10 R\right)-R$ is the gas constant, in $\mathrm{J} / \mathrm{mol} \mathrm{K}$ - are expected to exhibit crystal growth kinetics of the form predicted by the normal and screw dislocation growth models, respectively.

According to the normal model, the interface is rough on an atomic or molecular scale. Growth takes place at step sites intersecting the interface, and the growth rate, $U$, may be expressed by

$U=f \frac{D_{U}}{\lambda}\left[1-\exp \left(-\frac{\Delta G}{R T}\right)\right]$

where $D_{U}$ is an effective diffusion coefficient $\left(\mathrm{m}^{2} / \mathrm{s}\right)$ of the (unknown) species that controls atomic or molecular attachment at the interface; $\lambda$ is the (unknown) diameter of the diffusing building molecules ( $\mathrm{m}$ ), which is equivalent to the jump distance, the lattice parameter or the unit distance advanced by the interface; $\Delta G$ is the free energy change upon crystallization $(\mathrm{J} / \mathrm{mol}) ; T$ is the absolute temperature $(\mathrm{K})$, and $f$ is the fraction of preferred growth sites on the interface, that is close to unity.

For the screw dislocation model, $f \approx \frac{T_{\mathrm{m}}-T}{2 \pi T_{\mathrm{m}}}$ ( $T_{\mathrm{m}}$ is the melting temperature), the crystal-liquid interface is smooth, albeit imperfect on atomic scale, and growth takes place at step sites provided by screw dislocations. For brevity, the $2 D$ mechanism will not be considered here, but it could be found elsewhere [11].

To interpret experimental data with respect to the kinetic models described above, it is necessary to evaluate the diffusivity $D_{U}$. This parameter can be estimated with the Eyring equation, assuming that the molecular motions required for interfacial rearrangements controlling crystal growth is similar to those controlling viscous flow in the bulk liquid, $D_{U} \cong D_{\eta}$. Hence

$D_{\eta}=\frac{k_{\mathrm{B}} T}{\lambda \eta}$,

where $\eta$ is the shear viscosity (Pa s) and $k_{\mathrm{B}}$ is the Boltzmann constant. In general, the viscosity is expressed by means of the Vogel-Fulcher-Tammann-Hesse expression (VFTH) $\log _{10} \eta=A+\frac{B}{T-T_{0}}$, where $A, B$ and $T_{0}$ are constants.

It has been a matter of strong discussion if the Eyring equation can be used for calculations of crystal growth kinetics, especially at deep undercoolings, below $1.2 T_{\mathrm{g}}$, where it has been suggested that this equation fails (e.g.: see Refs. [9-11] and references cited therein). In this paper, the Eyring equation (Eq. (2)) is supposed to be valid from the melting point to $\approx 1.2 T_{\mathrm{g}}$, covering a wide temperature range that is of interest for viscous flow sintering. In such interval the VFTH expression is valid to describe viscous flow.

It is clear from the above discussion that one needs to know the glass viscosity as a function of temperature and other experimental parameters, such as the melting enthalpy $\Delta H_{\mathrm{m}}$ (or $\Delta G$ ) and $T_{\mathrm{m}}$, to compute crystal growth rates. At low undercoolings, $T \geqslant 0.85 T_{\mathrm{m}}$, the energy barrier, $\Delta G$, can be estimated by the Thomson/Turnbull (Eq. (3)):

$\Delta G=\frac{H_{\mathrm{m}}\left(T_{\mathrm{m}}-T\right)}{T_{\mathrm{m}}}$.

We will use this theoretical background to define the sinterability parameter.

\subsection{Viscous flow sintering}

In this part we briefly review and discuss two classical glass sintering models: Frenkel's and Mackenzie-Shuttleworth's, and then focus on the problem of viscous flow sintering with concurrent crystallization using the Clusters model.

\subsubsection{The Frenkel model $(F)$}

The Frenkel model [12] offers a description of the onset of isotropic sintering of monodispersed spherical particles. After a sintering time $t$, the linear shrinkage $(\Delta L)$ relative to the sample original length, $L_{0}$, is given by Eq. (4):

$\frac{\Delta L}{L_{0}}=\frac{3 \gamma}{8 \eta(T) r} t$

where $\eta(T)$ is the temperature-dependent shear viscosity, $\gamma$ is the glass-vapor surface energy (whose temperature dependence is very weak), and $r$ is the initial particle radius.

To describe the density change during sintering Eq. (5) is commonly used. In that equation, $\rho_{0}$ is the initial green density of the compact and $\rho_{g}$ is the actual density of the glass:

$\rho(T)=\frac{\rho_{0}}{\rho_{\mathrm{g}}}\left[1-\frac{3 \gamma t}{8 \eta(T) r}\right]^{-3}$

Deviations from Eq. (4) are found when the particles are jagged (such as crushed particles) [2-5]. To account for the effect of particle shape on the sintering kinetics, an empirical constant, denominated shape factor, $k_{\mathrm{S}}$, is normally used to fit the data. The $k_{\mathrm{S}}$ values used in the literature vary from 1.8 to 3 . Nevertheless, when one compares the sintering kinetics of spherical particles with that 
of irregular particles having the same size distribution, not only shape effect is being evaluated, but also the particle packing, that is different for different shapes. Thus, the real effect of the particles shape on the sintering kinetics is quite complex and deserves further attention.

The Frenkel equation, Eq. (4), was derived for a linear arrangement of particles. In passing to volume shrinkage, one can use Eq. (5). This passage assumes isotropic sintering in the three spatial coordinates, which is equivalent to consider a simple cubic array of particles. Thus, each particle should have six neighbors and, therefore, develop six sintering necks in the process. However, experimental data for an array of spherical glass particles having a narrow size distribution shows that a distribution of necks per particle (between 3 and 8) arises and that the average is about 5 [5]. This distribution varies for different systems and should, therefore, be tested case-by-case. An example is shown in Fig. 1(a).

\subsubsection{The Mackenzie-Shuttleworth model (MS)}

For higher relative densities $(\rho>0.9)$, when the pores are spherical and isolated in the glass, the Mackenzie-Shuttleworth model [13] gives the following densification rate:

$$
\frac{\mathrm{d} \rho(T)}{\mathrm{d} t}=\frac{3 \gamma}{2 a_{0} \eta(T)}(1-\rho),
$$

where $a_{0}$ is the initial radius of the spherical pores. Eq. (6) is presented here in a simplified form that allows for a simple mathematical treatment (for more details see Refs. [2-5]). We approximated the pore radius, $a(t)$, by the constant $a_{0}$, while the pore number remains fixed. This approximation slightly underestimates the actual sintering kinetics in the latest stages. Fig. 1(a) shows an example of application of this model.

\subsubsection{The Clusters model}

Giess et al. [14] reported that a pure MS analysis does not accurately describe the final stages of sintering of pressed compacts of polydispersed, irregular-shaped cordierite glass particles. He suggested that this drawback may be the result of small-size particles sintering most rapidly at the outset and large particles delaying sintering towards the end of the process. Such experimental evidence indicates that the F and MS stages may occur simultaneously in a sample having a particle size distribution.

The Clusters model [2] is based on this fact: small particles preferentially cluster in the open spaces left by larger particles and sinter faster. Thus, for a polydispersed compact of glass particles with volume fraction $v_{r}$ of particles of radius $r$, Eq. (7) holds true for the densification kinetics at a given temperature.

$\rho(t)=\frac{\sum_{r}\left[\rho_{\mathrm{F}}(r, t) \xi_{r} \theta\left(t_{0.8}-t\right)+\rho_{\mathrm{MS}}(r, t) \theta\left(t-t_{0.8}\right)\right] v_{r}}{\sum_{r}\left[\xi_{r} \theta\left(t_{0.8}-t\right)+\theta\left(t-t_{0.8}\right)\right] v_{r}}$.

Eq. (7) sums up the relative density $\rho(r, t)$ for each cluster having particle size $r$, as a function of time, $t$. During the Frenkel stage of sintering, the condition $\rho(r, t)=\rho_{\mathrm{F}}(r, t)<0.8$ is met and $\rho_{\mathrm{F}}(r, t)$ is calculated using the Frenkel model, Eq. (5). Later, $\rho(r, t)=\rho_{\mathrm{MS}}(r, t)>0.8, \rho_{\mathrm{MS}}(r, t)$ is calculated by the MackenzieShuttleworth model, Eq. (6), see Fig. 1(a). For each cluster, the passage from the $F$ to the MS regime is performed using the step function $\theta(x)$, which is unity for positive $x$ and null for negative $x$, thus alternating between 1 and 0 at $t=t_{0.8}$, when $\rho_{\mathrm{F}}\left(r, t_{0.8}\right)=0.8$ is reached. $\xi_{r}$ is the neck-forming ability of each particle having size $r$, which can be calculated from the particle size distribution. The empirical expression $\xi_{r}=1 / r^{c}$, where $c$ depends on the particle size distribution, is proposed in Ref. [2].

The pore radius $a_{0}$ in Eq. (6) is adjusted for each particle cluster to ensure a continuous $\rho(r, t)$ function at $t=t_{0.8}$. The adjustment is achieved by first computing $t_{0.8}$ with Eq. (5), then calculating $a_{0}$ with the integrated version of Eq. (6) at $t=t_{0.8}$, as shown in Fig. 1(a).

Eq. (7) can be explicitly written as below (Eq. (8) (for $\xi_{r}=1$, which corresponds to narrow particle size distribution:

$$
\begin{aligned}
\rho(t)= & \sum_{r}\left\{\frac{\rho_{0}}{\rho_{\mathrm{g}}\left[1-\frac{3 \gamma t}{8 \eta(T) r}\right]^{3}} \theta\left(t_{0.8}-t\right)+\theta\left(t-t_{0.8}\right)\left[1-\left(1-\frac{\rho_{0}}{\rho_{\mathrm{g}}}\right)\right.\right. \\
& \left.\left.\times \exp \left(-\frac{3 \gamma t}{2 a_{0} \eta(T)}\right)\right]\right\} v_{r}
\end{aligned}
$$

and an example is shown in Fig. 1(b).

Other aspects of the typical microstructure that should be considered in order to describe the sintering of actual glass particle compacts are:

(i) The number of necks that each particle develops with its neighbors: we have experimentally found [5] that the actual number of necks per particle in green compacts of monodispersed spheres varies from 3 to 8 necks/particle, with an average value of about 5 necks/particle.
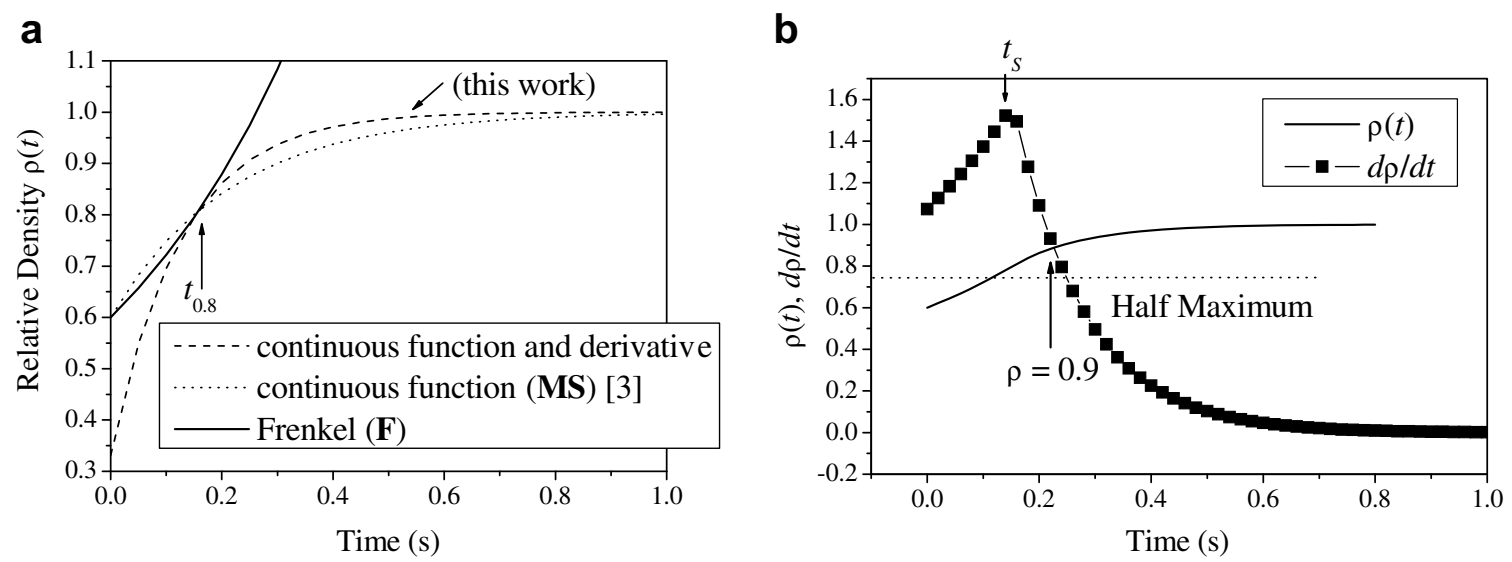

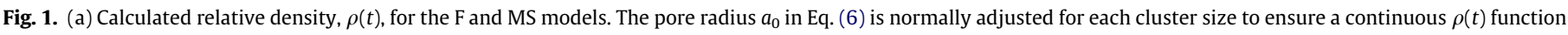

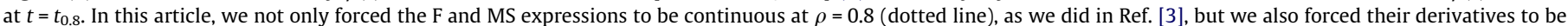

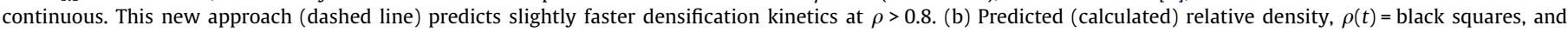
densification rate, $\mathrm{d} \rho(\mathrm{t}) / \mathrm{d} t=$ continuous line, for cordierite glass with $0.5 \mu \mathrm{m}$ particles at $1300 \mathrm{~K}$ ignoring crystallization. 
(ii) Particle surfaces with pre-existing solid inclusions: when the glass particles to be sintered show pre-existing crystals or dust on their surface, only the glass-glass contacts contribute to sintering. For example, particles having only $90 \%$ of glassy surface have an effective surface fraction $0.9 \times 0.9=0.81$ that is free for making contacts and developing necks during viscous flow sintering.

(iii) The assumed value $\rho_{r}=0.8$, for which Frenkel's model breaks down and the MS equation starts to prevail, is somewhat arbitrary and depends on the initial (green) packing density, $\rho_{0}$, of the powder compact. This value is fine for $\rho_{0}>0.6$. But an even better situation is found for $\rho_{0}>0.7$, when one can use $\rho_{r}=0.85$. So Eq. (8) is valid for $\rho_{0}>0.6$.

\subsection{The proposed sinterability parameter, $S$}

\subsubsection{Times to achieve the maximum sintering $\left(t_{S}\right)$ and crystallization $\left(t_{C}\right)$ rates}

For simplicity, in the following paragraphs we only analyze isothermal sinter-crystallization processes. Thus we assume that the heating rate of the glass powder compact is fast enough to avoid surface crystallization on the heating path and that both sintering and surface crystallization occurs only during the isothermal treatment. With this condition, at any given sintering temperature, time is the key variable. Full densification occurs if the time period necessary to fully sinter any given glass powder compact is less than the time for crystallization to start.

In order to pictorially understand the proposed method to find a characteristic time for each of these two processes (densification and crystallization), let us analyze Figs. 1(a), (b) and 2. Fig. 1(b) shows the sintering kinetics corresponding to $0.5 \mu \mathrm{m}$ cordierite glass particles calculated from Müller's [7] data for $\eta(T), U(T)$, and $N_{\mathrm{S}}$ using the Clusters model [3]. In Ref. [3], the compact's sintering kinetics was calculated by the Frenkel model (F) up to a relative density $\rho_{r}=0.8$, and afterwards by the Mackenzie-Shuttleworth (MS) model. At $\rho_{r}=0.8$ the MS pore size was conveniently chosen to make the compact's density function continuous. In this article, we not only forced the F and MS expressions to be continuous at $\rho=0.8$ (as we did in Ref. [3]), but we also forced their derivatives to be continuous because later on in this paper we need to calculate the derivative $\frac{\partial \rho}{\partial t}$ for each time during densification. As shown in Fig. 1(a), this new approach predicts slightly faster densification kinetics at $\rho>0.8$ than the approach of Ref. [3]. Nevertheless, in both cases the time required to achieve the maximum sintering rate corresponds to the time when the compact's density is $\rho=0.8$.

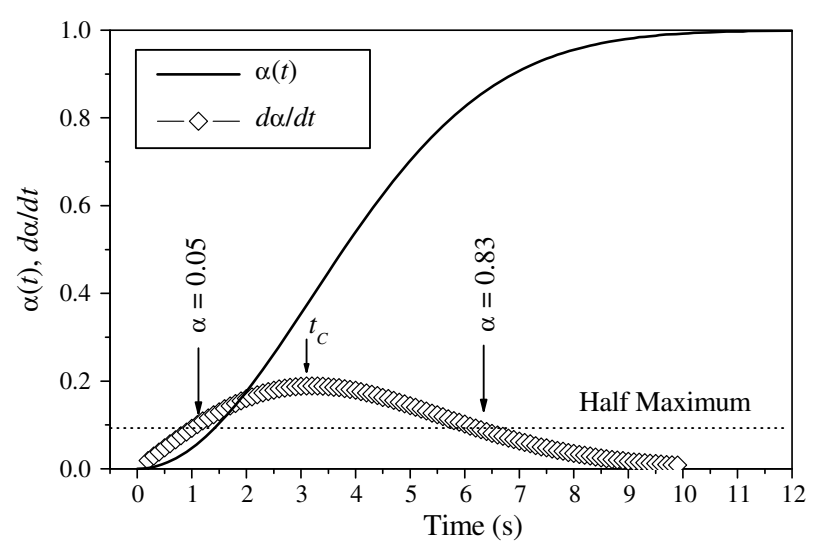

Fig. 2. Crystallized surface fraction, $\alpha(t)$, and surface crystallization rate, $d \alpha(t) / d t$, for cordierite glass particles at $1300 \mathrm{~K}$.
Fig. 1(a) shows the individual contributions of the $\mathrm{F}$ and MS stages to the sintering curve. The time corresponding to the maximum sintering rate, $t_{\mathrm{S}}$, is thus well defined. If crystallization is taken into account in the sintering calculations (using the Clusters model), $t_{\mathrm{S}}^{\mathrm{C}}$ is typically larger than its value for viscous sintering without crystallization, $t_{\mathrm{S}}$. However, for relatively large particle sizes, $t_{\mathrm{S}}$ could be shorter than $t_{\mathrm{S}}^{\mathrm{C}}$, since crystallization arrests sintering in its first stages (i.e., before a relative density of 0.8 is reached). Fig. 2 shows that also in this case a certain time is necessary for the maximum crystallization rate, $t_{\mathrm{C}}$, to occur.

For simplicity, we calculate the sintering time without crystallization, $t_{\mathrm{S}}$, and study the sinter-crystallization concurrence by analyzing how close are $t_{\mathrm{S}}$ and $t_{\mathrm{C}}$. This difference will lead to a sinterability parameter. We then test the derived parameter against a rigorous numerical simulation which takes into account densification with concurrent crystallization.

\subsubsection{The relative positions of $t_{S}$ and $t_{C}$}

To illustrate our ideas, Fig. 3 shows that at $1300 \mathrm{~K}, 0.5 \mu \mathrm{m}$ cordierite particles sinter up to full density $\rho=1.0$ (despite some surface crystallization), but the densification of $6 \mu \mathrm{m}$ particles saturates at a relative density of 0.91 . In the former case only a few percent of the particle surfaces crystallized when the compact reached full density, however, almost $70 \%$ of the surface of the $6 \mu \mathrm{m}$ particles were crystallized when a relative density of 0.9 was reached. For this specific case, the inset of Fig. 3 shows that for the $6 \mu \mathrm{m}$ particles, the times $t_{\mathrm{S}}$ corresponding to the maximum sintering rates, $\mathrm{d} \rho /\left.\mathrm{d} t\right|_{\max }$, are approximately the same, with or without considering the effect of crystallization.

Fig. 4 shows that the relative position of the densification maximum, $t_{\mathrm{S}}$, significantly changes with particle size, thus the relative positions of $t_{\mathrm{S}}$ and $t_{\mathrm{C}}$ can be good indicators of the concurrence between sintering and crystallization.

\subsubsection{Calculation of $t_{C}$}

In the case of isothermal surface crystallization of spherical crystals (circles on the glass particle surfaces) from a fixed number of nucleation sites, $N_{\mathrm{S}}$, with constant growth rate $U(T)=$ constant, the crystallized surface fraction is given by the Johnson-MehlAvrami-Kolmogorov (JMAK) expression:

$\alpha(t)=1-\exp \left(-\pi N_{S}[U(T) t]^{2}\right)$.

To find out $t_{c}$, the time at which the crystallization rate is maximum, we take the second derivative of Eq. (9) with time and equate it to zero to give

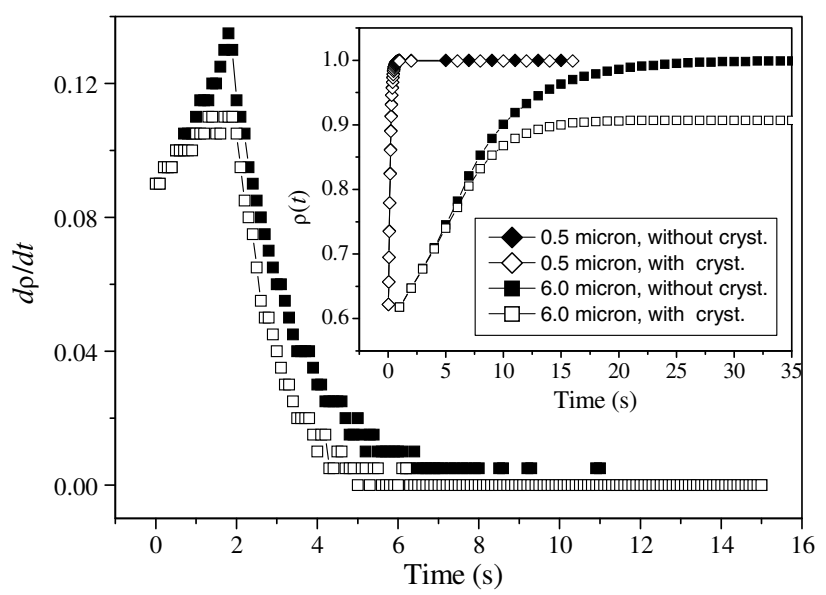

Fig. 3. Sintering rates of $6 \mu \mathrm{m}$ particles without ( $\mathbf{\square})$ and with $(\square)$ crystallization. Inset: Densification curve of 0.5 and $6 \mu \mathrm{m}$ particles at $1300 \mathrm{~K}$, with (open symbols) and without (closed symbols) the arresting effect of crystallization. 


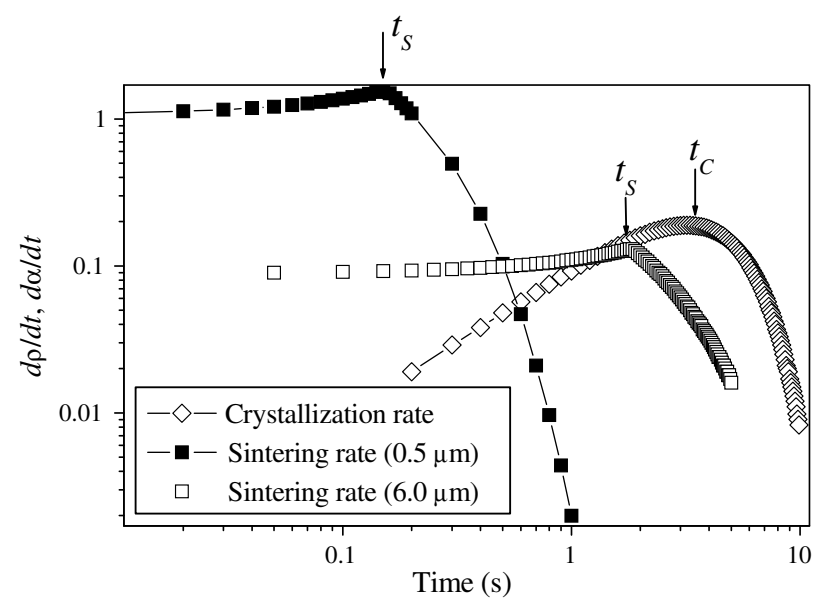

Fig. 4. Sintering and crystallization rates of cordierite glass particles of 0.5 and $6.0 \mu \mathrm{m}$ at $1300 \mathrm{~K}: \alpha(t)$ is the crystallized surface fraction, and $\rho(t)$ the relative density of the compact without crystallization.

$$
t_{\mathrm{C}}=\frac{1}{\sqrt{2 \pi N_{\mathrm{S}}} U(T)} \text {. }
$$

But a close look at Fig. 4 suggests that we should also consider the peak widths. In other words, if both sintering and crystallization peaks in Fig. 4 were Dirac-delta functions, it would only be necessary to determine which one is located at the lowest temperature. Then, while passing that temperature in a heating experiment, the respective process (sintering or crystallization) would be completed. In practice, however, sintering and crystallization take place along a time interval, and the more the sintering time interval overlaps with the crystallization time interval, the fiercer will be the competition between these phenomena.

Therefore, we numerically determined that at times corresponding to $\alpha=0.05$ and $\alpha=0.843$ the crystallization rates are half their maximum rate (see Fig. 2). Using Eq. (9), with $\alpha=0.05$ and $\alpha=0.843$, the width of the crystallization peak $\left(\Delta_{\mathrm{C}}\right)$ can thus be estimated as

$\Delta_{\mathrm{C}}=\frac{\sqrt{-\ln (1-0.843)}-\sqrt{-\ln (1-0.05)}}{\sqrt{2 \pi N_{\mathrm{S}}} U(T)} \approx \frac{2}{3 \sqrt{N_{\mathrm{S}}} U(T)}$.

Although the crystallization rate curve is not symmetric, to a first approximation we estimate the half-width as

$\frac{\Delta_{\mathrm{C}}}{2}=\frac{1}{3 \sqrt{N_{\mathrm{S}}} U(T)}$.

\subsubsection{Calculation of $t_{S}$}

To obtain a reliable value for $t_{\mathrm{s}}$, first of all we need a good model for the sintering kinetics. The calculation of the sintering kinetics will depend on the system under consideration. For example, for gels and other loose packed systems, Scherer's model $[1,15]$ should be used.

For glass particle compacts of micron size or larger particles, starting with a green density $\rho_{0}=0.6$ or larger, the sintering kinetics is well described by the Clusters model [2-6]. In this model, packed monodispersed particles with $\rho_{0} \geqslant 0.6$ sinter up to $\rho=0.8$ with kinetics given by the Frenkel model, and afterwards by the MS model, as explained above. Within the framework of the Clusters model, the maximum sintering rate is reached at $\rho \approx 0.8$, since the MS assumes a lower sintering rate. From the $\mathrm{F}$ model, the $t_{\mathrm{S}}$ value at $\rho=0.8$, with $\rho_{0}=0.6$ is

$t_{\mathrm{S}}=\sqrt[3]{1-\frac{\rho_{0}}{\rho}} \frac{8 \eta r}{3 \gamma} \approx 1.7 \frac{\eta r}{\gamma}$
Since the sintering curve is highly asymmetric, it is not trivial to define a width. However, as viscous sintering proceeds over a certain period of time, we need to estimate its duration. To simplify the derivations, we will take the typical case of $\rho_{0}=0.6$, and consider the width as twice the time required to sinter from a relative density 0.7 to 0.8 :

$\Delta_{\mathrm{S}}=2\left[\sqrt[3]{1-\frac{\rho_{0}}{0.8}}-\sqrt[3]{1-\frac{\rho_{0}}{0.7}}\right] \frac{8 \eta r}{3 \gamma} \approx 0.6 \frac{\eta r}{\gamma}$.

Therefore, just to gauge its magnitude, we will use the halfwidth of the sintering rate curve as

$\frac{\Delta_{\mathrm{s}}}{2}=0.3 \frac{\eta r}{\gamma}$.

\subsubsection{The sinterability parameter, $S$}

Fig. 4 shows that the separation of the sintering and crystallization rate peaks, taking into account their respective half-widths, can be estimated by the difference $\left(t_{\mathrm{C}}-\frac{\Delta_{\mathrm{C}}}{2}\right)-\left(t_{\mathrm{S}}+\frac{\Delta_{\mathrm{S}}}{2}\right)$. The larger the value of this difference, the smaller is the overlapping between sintering and crystallization, and thus the larger will be the sinterability.

We then propose that a necessary condition for sinterability is

$\left(t_{\mathrm{C}}-\frac{\Delta_{\mathrm{C}}}{2}\right)-\left(t_{\mathrm{S}}+\frac{\Delta_{\mathrm{S}}}{2}\right)>0$

Substituting Eqs. (10), (12), (13), and (15) into Eq. (16) we get an equivalent condition:

$\frac{\gamma}{\sqrt{N_{S}} U \eta r}>10$.

We shall thus denominate sinterability, $S$, the following adimensional parameter:

$S=\frac{\gamma}{\sqrt{N_{S}} U \eta r}$

and will use it to evaluate the degree of densification of different glass-forming systems that exhibit predominant surface crystallization. Such equation shows that any glass-forming system with high viscosity, high crystal growth rates or low $\gamma$ is hard to densify. Thus, according to Eqs. (17) and (18), for $S>10$ any crystallizing glass powder can (theoretically) be densified before crystallization up to a given, unknown density value. Later in this article we will empirically demonstrate that a glass-forming system must have $S>50$ to reach a relative density $>0.99$. This result does not contradict the prediction of Eqs. (17) and (18).

\subsubsection{Derivation of an expression for the product $U \cdot \eta$}

In this section we will derive an expression to estimate the product $U \cdot \eta$ in Eq. (18). The idea is to find a way to avoid the (time consuming) measurement of crystal growth rates and viscosities. To accomplish this task we will use available viscosity and crystal growth rate data in a wide temperature range for several silicate and borate glass-forming systems (Table 1), see details in Refs. [16-18].

From Eqs. (1) and (3), for the well-known normal or screw dislocation growth models, considering low undercoolings, one has

$U \cdot \eta \approx \frac{f}{N_{\mathrm{A}}} \frac{\Delta T}{T_{\mathrm{m}}} \frac{\Delta H_{\mathrm{m}}}{\sqrt[3]{V_{\mathrm{m}}^{2}}}$

where $f=1$ for normal or $f(T)=\frac{\Delta T}{2 \pi T_{\mathrm{m}}}$ for screw dislocation growths, respectively; $V_{m}$ is the molar volume and $\Delta T=T-T_{\mathrm{m}}$ is the undercooling. A test of Eq. (19) is presented below. 
Table 1

Thermodynamic, viscosity and crystal growth rate data for the selected glass-forming systems used here

\begin{tabular}{|c|c|c|c|c|c|c|c|c|c|c|c|}
\hline Glass & $A$ & $B(\mathrm{~K})$ & $T_{0}(\mathrm{~K})$ & Growth $^{*}$ mechanism & $\Delta T$ range $(\mathrm{K})$ & $\Delta H_{\mathrm{m}}(\mathrm{kJ} / \mathrm{mol})$ & $T_{\mathrm{g}}(\mathrm{K})$ & $T_{\mathrm{m}}(\mathrm{K})$ & $V_{\mathrm{m}}\left(\mathrm{cm}^{3} / \mathrm{mol}\right)$ & $\gamma^{* *}\left(\mathrm{~J} / \mathrm{m}^{2}\right)$ & Reference \\
\hline $\mathrm{Li}_{2} \mathrm{O} \cdot 2 \mathrm{SiO}_{2}$ & -2.623 & 3388.8 & 491.0 & SD & $0.98 T_{\mathrm{g}}-T_{\mathrm{m}}$ & 57.3 & 727 & 1306 & 61.5 & 0.320 & [16-18] \\
\hline $\mathrm{Na}_{2} \mathrm{O} \cdot 2 \mathrm{SiO}_{2}$ & -3.075 & 4595.8 & 392.9 & SD & $1.16 T_{\mathrm{g}}-T_{\mathrm{m}}$ & 33.5 & 728 & 1146 & 70.9 & 0.292 & [16-18] \\
\hline $\mathrm{Na}_{2} \mathrm{O} \cdot 3 \mathrm{SiO}_{2}$ & -2.687 & 4451.4 & 427.5 & SD & $1.04 T_{\mathrm{g}}-T_{\mathrm{m}}$ & 36.05 & 743 & 1084 & 73.53 & 0.227 & [16-18] \\
\hline $\mathrm{K}_{2} \mathrm{O} \cdot 2 \mathrm{SiO}_{2}$ & -5.00 & 7460.54 & 332.67 & SD & $1.25 T_{\mathrm{g}}-T_{\mathrm{m}}$ & 31.8 & 768 & 1313 & 84.7 & 0.210 & [16-18] \\
\hline $\mathrm{CaO} \cdot \mathrm{MgO} \cdot 2 \mathrm{SiO}_{2}$ & -4.27 & 3961.2 & 750.9 & SD & $1.05 T_{\mathrm{g}}-T_{\mathrm{m}}$ & 138 & 995 & 1664 & 75.9 & 0.366 & [11] \\
\hline $2 \mathrm{MgO} \cdot 2 \mathrm{Al}_{2} \mathrm{O}_{3} \cdot 5 \mathrm{SiO}_{2}$ & -3.97 & 5316 & 762 & SD & $0.99 T_{\mathrm{g}}-T_{\mathrm{m}}$ & 180 & 1088 & - & 112.3 & 0.360 & {$[6,17]$} \\
\hline $\mathrm{LAS}_{2}$ & -3.34 & 5162.0 & 511.8 & ${ }^{* * *}$ & $1200-T_{\mathrm{m}}$ & 38 & - & 1653 & - & 0.421 & [21] \\
\hline NBS & -3.33 & 4302.0 & 545.0 & No cryst. & $873-1100$ & - & - & - & - & 0.240 & [22] \\
\hline $\mathrm{Li}_{2} \mathrm{O} \cdot 2 \mathrm{~B}_{2} \mathrm{O}_{3}$ & -4.951 & 2466.8 & 617.3 & SD & $1.04 T_{\mathrm{g}}-T_{\mathrm{m}}$ & 120.5 & 763 & 1190 & 69.31 & $0.182^{* * * *}$ & [16-18] \\
\hline $\mathrm{Na}_{2} \mathrm{O} \cdot 2 \mathrm{~B}_{2} \mathrm{O}_{3}$ & -3.8956 & 1909.5 & 621.6 & SD & $1.15 T_{\mathrm{g}}-T_{\mathrm{m}}$ & 75.7 & 748 & 1015 & 84.9 & 0.157 & [16-18] \\
\hline
\end{tabular}

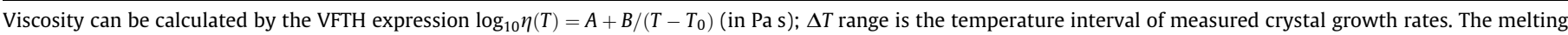

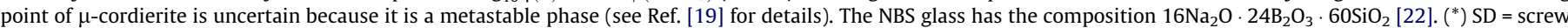

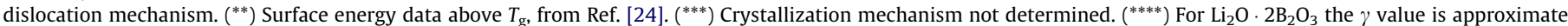
due to lack of experimental data, and refers to $37 \mathrm{Li}_{2} \mathrm{O} \cdot 63 \mathrm{~B}_{2} \mathrm{O}_{3} \mathrm{~mol} \%$ composition.

\section{Results}

Let us first test Eq. (19) against experimental data for the product $U(T) \cdot \eta(T)$ using the glass-forming systems listed in Table 1 (Figs. 5(a)-(h)). For approximate fits with Eq. (19), we considered $f=f(T)$, dashed line (please check Ref. [16] for details on data mining of $U$ and Refs. $[17,18]$ for $\eta$ ). Fig. 5 shows a fast decrease of $U \cdot \eta$ with temperature, starting at about $T_{\mathrm{g}}$, reaching a near constant region, which stays up to the temperature of maximum crystal growth rate, $U_{\max }$, with further decrease until $T_{\mathrm{m}}$ is reached, as expected because, by definition, $U\left(T_{\mathrm{m}}\right)=0$.

Below we describe experimental tests of Eq. (19) performed with several stoichiometric glass-forming systems:

\subsection{Lithium disilicate glass}

Lithium disilicate $\left(\mathrm{Li}_{2} \mathrm{O} \cdot 2 \mathrm{SiO}_{2}\right)$ glass is one of the most studied stoichiometric glass-forming systems, with a plethora of data for $U$ and $\eta$ [16]. The experimental $U \cdot \eta$ for such system is shown in Fig. 5(a) to be in good agreement with the calculations by the approximate screw dislocation model (Eq. (19)) for temperatures above $0.85 T_{\mathrm{m}}$.

\subsection{Sodium disilicate glass}

For the sodium disilicate $\left(\mathrm{Na}_{2} \mathrm{O} \cdot 2 \mathrm{SiO}_{2}\right)$ glass used here, $U(T)$ and $\eta(T)$ were determined for samples of the same batch and this is the best situation one can think. The experimental product $U \cdot \eta$ follows the curve calculated by the approximate screw dislocation model for temperatures above $0.89 T_{\mathrm{m}}$ (Fig. 5(b)).

\subsection{Sodium trisilicate glass}

For sodium trisilicate $\left(\mathrm{Na}_{2} \mathrm{O} \cdot 3 \mathrm{SiO}_{2}\right)$ glass, the experimental $U \cdot \eta$ shows excellent agreement with the predictions of Eq. (19) using the approximate screw dislocation model. The limit of validity is $T>0.80 T_{\mathrm{m}}$ (Fig. 5(c)).

\subsection{Potassium disilicate glass}

According to Fig. 5(d), for potassium disilicate $\left(\mathrm{K}_{2} \mathrm{O} \cdot 2 \mathrm{SiO}_{2}\right)$ glass there is good agreement of experimental and calculated $U \cdot \eta$ via the approximate screw dislocation model (Eq. (19)) for $T>0.84 T_{\mathrm{m}}$.

\subsection{Diopside glass}

Experimental data of $U(T)$ and $\eta(T)$ for diopside (CaO . $\mathrm{MgO} \cdot 2 \mathrm{SiO}_{2}$ ) glass in a wide temperature range was recently collected [11]. In this case the approximate screw dislocation growth expression describes the experimental $U \cdot \eta$ data above $0.93 T_{\mathrm{m}}$ (Fig. 5(e))

\subsection{Cordierite glass}

Relevant data to calculate the product $\eta \cdot U$ for cordierite $\left(2 \mathrm{MgO} \cdot 2 \mathrm{Al}_{2} \mathrm{O}_{3} \cdot 5 \mathrm{SiO}_{2}\right)$ glass was taken from Ref. [19]. The approximate screw dislocation model gives good agreement with experiment for temperatures above $0.80 T_{\mathrm{m}}$ (Fig. 5(f)).

\subsection{Lithium diborate glass}

For $\mathrm{Li}_{2} \mathrm{O} \cdot 2 \mathrm{~B}_{2} \mathrm{O}_{3}$ Eq. (19) based on the approximate screw dislocation growth model, gives reasonable agreement with the experimental $U \cdot \eta$ above $0.82 T_{\mathrm{m}}$ (Fig. $5(\mathrm{~g})$ ).

\subsection{Sodium diborate glass}

For $\mathrm{Na}_{2} \mathrm{O} \cdot 2 \mathrm{~B}_{2} \mathrm{O}_{3}$ glass the agreement between experimental and calculated $U \cdot \eta$ using the approximate screw dislocation expression is reasonable above $0.92 T_{\mathrm{m}}$ (see Fig. $5(\mathrm{~h})$ ).

\section{Discussion}

In the following paragraphs we derive a simpler expression for prediction of sinterability at high temperatures that avoids the measurement of $U(T)$ and $\eta(T)$, then test both the original and the approximate sinterability parameters for several glass-forming systems.

Figs. 5(a)-(h) show that for high temperatures, $T \geqslant 0.85 T_{\mathrm{m}}$, the simpler expression, Eq. (19), can be used to predict the product $U \cdot \eta$ based on easily determined thermodynamic properties, such as melting temperature, $T_{\mathrm{m}}$, enthalpy of fusion, $\Delta H_{\mathrm{m}}$, and molar volume, $V_{\mathrm{m}}$. The sinterability parameter $S(T)$ - Eq. (18) - is inversely proportional to the product $U(T) \cdot \eta(T)$, but such product can be represented by a simplified form if one considers the normal or screw dislocation growth mechanism for $U(T)$, according to Eq. (19). Therefore, by replacing Eq. (19) into Eq. (18) one has

$S_{h T}(T)=\frac{2 \pi \cdot \gamma \cdot N_{\mathrm{A}} \cdot T_{\mathrm{m}}^{2} \cdot \sqrt[3]{V_{\mathrm{m}}^{2}}}{10 \sqrt{N_{\mathrm{S}}} \cdot r \cdot \Delta H_{\mathrm{m}} \cdot \Delta T^{2}}$

This approximate expression for the sinterability parameter is only valid at low undercoolings, but avoids time consuming measurements of $U(T)$ and $\eta(T)$.

Using available data for cordierite, we calculated $S$ values for different particle sizes by Eq. (18). Independently, using the Clusters model (which takes the arresting effect of surface crystallization into account) we calculated the final (saturation) density, 

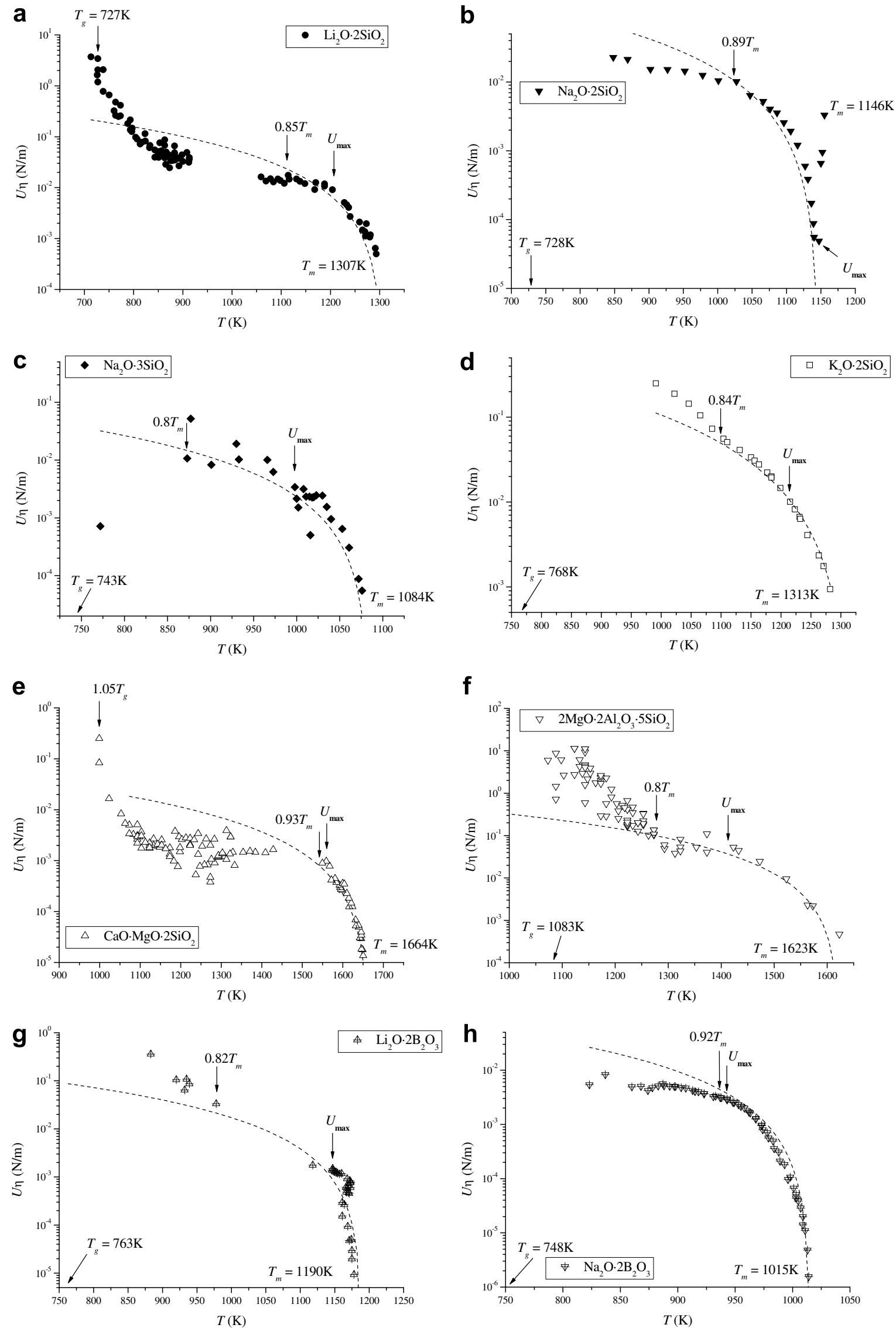

Fig. 5. Comparison of $U \cdot \eta$ calculated by Eq. (19) - dashed lines - and experimental data for the glass-forming systems shown in Table 1. 
$\rho_{\max }$, for the same conditions. We also fixed $r$ and varied the temperature, and thus independently calculated $\rho_{\max }$ and $S$ to check whether there was any relationship between these two parameters. In this way we generated Table 2 using real physicochemical data for a cordierite glass.

As a semi-quantitative test for $S$, we expect that the saturation densities of a power compact will be larger for systems with a large $S$. Indeed, Table 2 shows that the higher the value of the sinterability parameter, the higher the saturation density of the glass compact corroborating the validity of $S$ for a simple estimate of sinterability of glass powders.

We calculated the saturation density of glass powder compacts sintered at different temperatures with some data of Table 1 and the Clusters model [3], assuming $1 \mu \mathrm{m}$ particles, $r=10^{-6} \mathrm{~m}$, and a typical number of nucleation sites, $N_{\mathrm{S}}=10^{10} \mathrm{~m}^{-2}$, for all glasses. For each glass, we determined the temperature interval at which the saturation density $\rho_{\max }$ was larger than the arbitrary value of 0.99 . These temperature regions were then highlighted with solid symbols in Fig. 6 . When we marked these temperature regions in Fig. 7, we observed that $S>50$ is necessary for any glass power to reach a relative density larger than 0.99 in isothermal experiments. Therefore, $S>50$ is a necessary and sufficient condition for good sinterability.

In the next section an analysis of the sinterability parameter for different glasses is made using particle sizes of $1 \mu \mathrm{m}$ and $N_{\mathrm{S}}=10^{10} \mathrm{~m}^{-2}$. In this way the only variable quantities were the intrinsic physical properties of the glasses. The results are shown in Fig. 7 and discussed below.

(a) $\mathrm{SiO}_{2}$ is an Arrhenian glass. The transport properties of this specific glass suffer very strong effects from impurities. Sintering data for our calculations were taken from Ref. [20] for

Table 2

Sinterability of cordierite glass as a function of particle size and temperature

\begin{tabular}{llll}
\hline$T(\mathrm{~K})$ & $r(\mu \mathrm{m})$ & $\rho_{\max }$ & $S$ \\
\hline 1250 & 1 & 0.995 & 56.8 \\
1250 & 3 & 0.96 & 18.9 \\
1300 & 6 & 0.91 & 10.7 \\
1250 & 6 & 0.89 & 9.5 \\
1200 & 6 & 0.84 & 6.8 \\
1170 & 6 & 0.80 & 4.9
\end{tabular}

$S$ was calculated by Eq. (17) and $\rho_{\max }$ by the Clusters model.

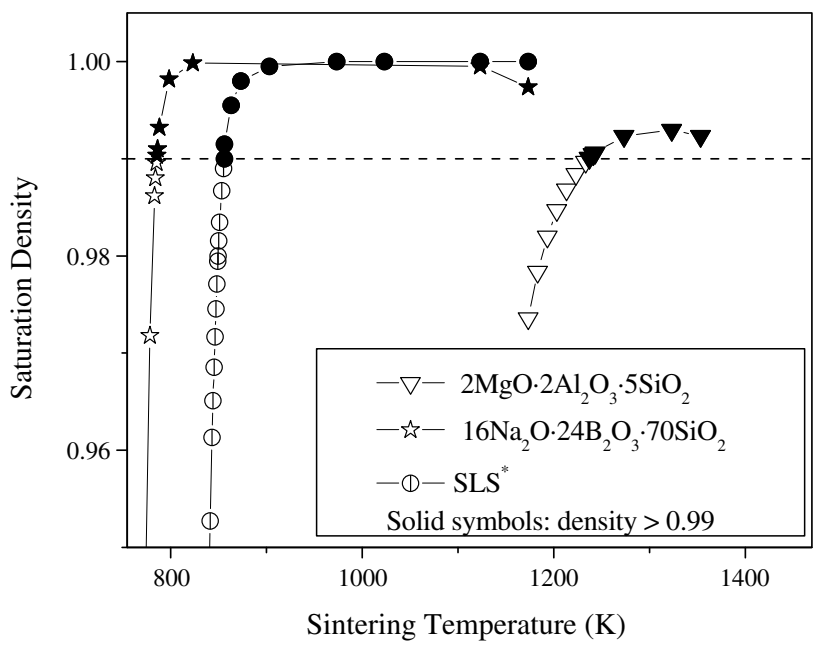

Fig. 6. Saturation density of glass powder compacts as a function of sintering temperature. (Saturation density is the maximum relative density reached by a compact when heated for a sufficiently long time at a temperature $T$ ).

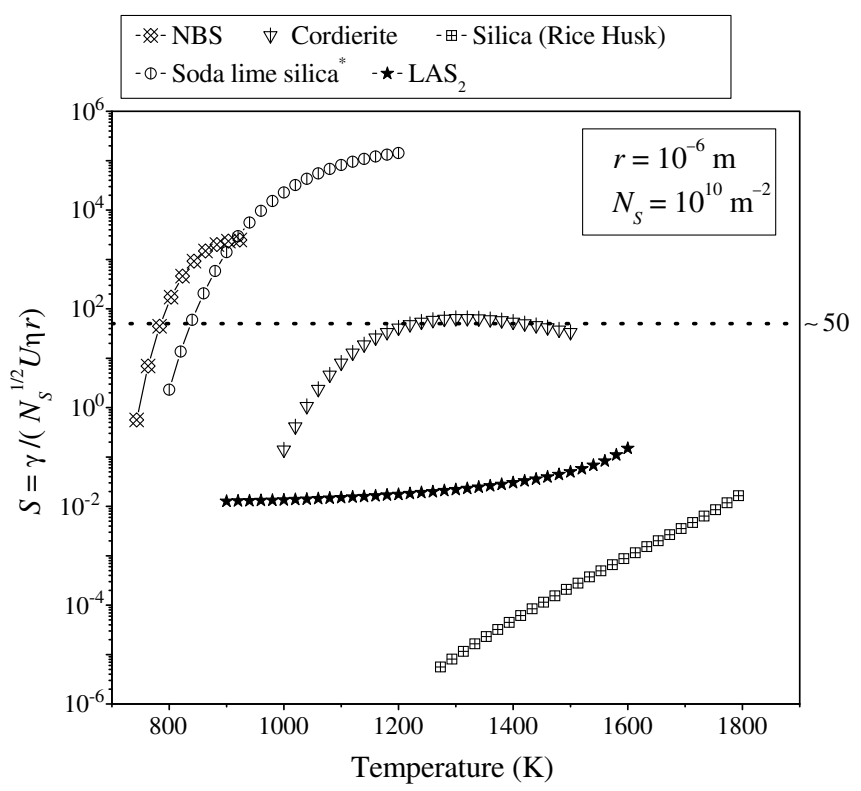

Fig. 7. Comparison of sinterability parameters $S$ using experimental $U$ and $\eta$ for different glasses as a function of temperature $\left(r=1\right.$ micron and $\left.N_{\mathrm{S}}=10^{10} \mathrm{~m}^{-2}\right)$. The horizontal line at $S \approx 50$ means that all systems reached a relative density $>0.99$.

a (impure) silica glass powder derived from rice hush ash. The viscosity of this particular sample was $\log _{10} \eta=-5.88+19679 / T$ ( $T$ in $\mathrm{K}, \eta$ in Pa s). The $S$ parameter is very low at the analyzed temperatures indicating a great difficult to sinter such powder, and this is due to the high crystal growth rates in this particular silica glass. A simulation with the Clusters model indicates that $1 \mu \mathrm{m}$ particles are only sinterable at high temperatures, above $1750 \mathrm{~K}$, but only if a very low $N_{\mathrm{S}}$, approximately $10^{2} \mathrm{~m}^{-2}$, is used in the calculations.

(b) Soda-lime silica glass is very complex for sintering studies because it shows at least three crystal phases (devitrite, cristobalite and wollastonite). For one particular composition with $72.5 \mathrm{SiO}_{2}, 13.7 \mathrm{Na}_{2} \mathrm{O}, 9.8 \mathrm{CaO}, 3.3 \mathrm{MgO}, 0.4 \mathrm{Al}_{2} \mathrm{O}_{3}, 0.2$ $\mathrm{FeO} / \mathrm{Fe}_{2} \mathrm{O}_{3}, 0.1 \mathrm{~K} 2 \mathrm{O}$ (wt\%), the VFTH expression used was $\log _{10} \eta=-2.7+4358.4 /(T-533.2), T$ in $\mathrm{K}, \eta$ in Pa s. A good temperature range for isothermal sintering is expected to start at $840 \mathrm{~K}$.

(c) For cordierite, the melting point of the metastable phase is unknown, but is between 1350 and $1467^{\circ} \mathrm{C}$ (see Ref. [19] for details)). Using the above described conditions for $N_{S}$ and $r$ and using $\eta$ and $U$ from Refs. [6,19], the expected optimum temperature range for isothermal sintering starts at $1220 \mathrm{~K}$.

(d) The sinterability parameter for $\mathrm{LAS}_{2}$ glass is below one, even for $1 \mu \mathrm{m}$ particles and high temperatures (Fig. 7). This is because this system has very high crystal growth rates that impair sintering, as it has been experimentally observed [21].

(e) An optimum temperature range for isothermal sintering of the sodium borosilicate glass at $928 \mathrm{~K}$ is expected (data of $\eta$ and $U$ are from Ref. [22]). From Fig. 7 it is possible to note that $1 \mu \mathrm{m}$ particles sinter well over all temperature range studied.

An important assumption in our calculations is that the heating rate is always fast enough to avoid surface crystallization on the heating path and that everything occurs in the sintering treatment, i.e., we considered an isothermal process at each temperature.

The approximations used to calculate $S_{h t}$ without experimental data on $U(T)$ and $\eta(T)$ - restrict its applicability to low undercoo- 


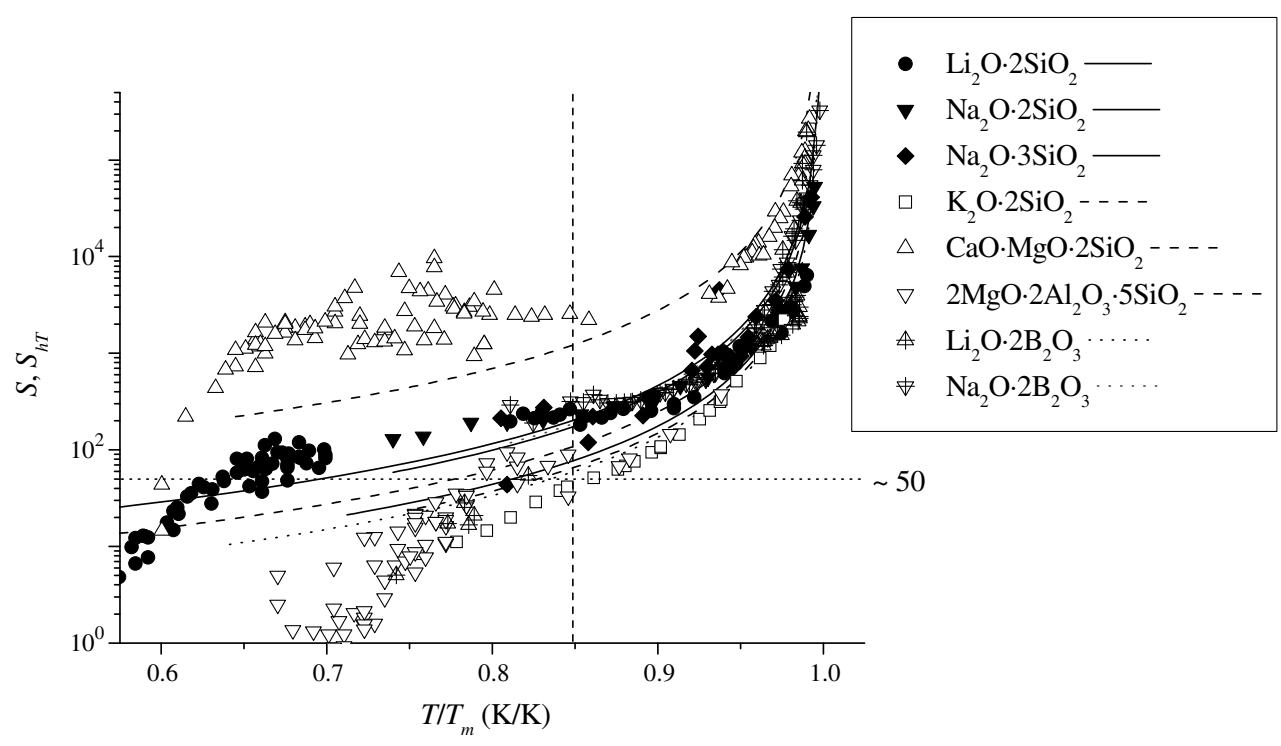

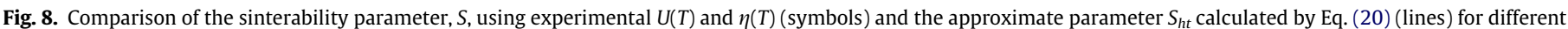

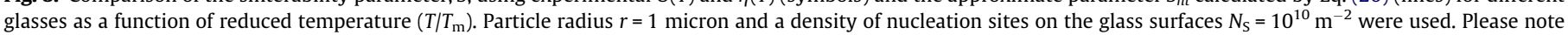
that $S_{h t}$ is approximately valid for temperatures higher than $0.85 T_{\mathrm{m}}$ for all glasses.

lings, i.e., to temperatures above $\approx 0.85 T_{\mathrm{m}}$, using the screw dislocation $[f=f(T)]$ or normal growth models $[f=1]$. But, by considering experimental crystal growth and viscosity data (i.e., with $S$ instead of $S_{h T}$ ) it is possible to predict the sinterability for much wider undercoolings, from the melting point down to $T_{\mathrm{g}}$.

For any given system, the glass/vapor surface energy only slightly depends on temperature, $N_{\mathrm{S}} \sim 10^{10} \mathrm{~m}^{-2}$ is a reasonable value for crushed glass particles [23], and the average particle size, $r$, can be treated as a variable. Therefore the only remaining variables to calculate $S$ or $S_{h t}$ are $U(T)$ and $\eta(T)$ or the parameters of the approximate expression, Eq. (19). Hence, $S$ will be highest for the lowest values of the product $U(T) \cdot \eta(T) r$.

One word of caution is necessary here: entrapped gases in the closing pores or crystallization induced degassing, which are not taken into account in the present analysis, may severely impair densification in the final stages of sintering. Therefore, a few percent residual (closed) porosity is frequently observed in sintered glass or glass-ceramic pieces.

\section{Conclusions}

We propose a parameter $S(T)=\gamma /\left\lfloor\sqrt{N_{S}} \cdot U(T) \cdot \eta(T) \cdot r\right\rfloor$ to gauge the sinterability of crystallizing glass powders without doing any sintering experiment. This parameter was tested for cordierite glass and the results show a strong correlation of $S$ with the maximum achievable densification for a series of particle sizes and temperatures. But a simpler expression:

$S_{h T}(T)=2 \pi \cdot \gamma \cdot N_{\mathrm{A}} \cdot T_{\mathrm{m}}^{2} \cdot \sqrt[3]{V_{\mathrm{m}}^{2}} / 10 \sqrt{N_{\mathrm{S}}} \cdot r \cdot \Delta H_{\mathrm{m}} \cdot \Delta T^{2}$,

which avoids time consuming measurements of $U(T)$ and $\eta(T)$, can be used at $T \geqslant 0.85 T_{\mathrm{m}}$ to predict the sintering behavior of glass powders.

The condition to densify any crystallizing glass powder to at least 0.99 is $S>50$. To privilege sintering over surface crystallization for any given glass, one must increase $S$ by minimizing $r$, or the product $U(T) \cdot \eta(T)$ by increasing the sintering temperature. $S$ increases with temperature and tends to infinity as the melting point of the crystal or liquidus is approached. Therefore, as long as the heating rate is fast enough to avoid crystallization on the heating path, and in the absence of entrapped gasses or degassing, any glass powder can be fully densified at a sufficiently high temperature, especially near and above the liquidus. But too high temperatures lead to excessive flow and deformation of the compact.

The main finding is that one can make predictions with $S$ without performing any sintering experiment. This parameter is thus quite useful for screening candidate glass compositions for sinter crystallization studies and for the production of sintered glasses or glass-ceramics (Fig. 8).

\section{Acknowledgments}

We thank the Comisión Nacional de Energía Atômica - CNEA (Argentina), Project 070/04 CAPES-SECYT, Fundação de Amparo à Pesquisa do Estado de São Paulo - FAPESP(Brazil), Contract nos. 04/ 10703-0 and 07/08179-9, and Conselho Nacional de Desenvolvimento Científico e Tecnológico - CNPq (Brazil) for funding this research.

\section{References}

[1] G.W. Scherer, J. Sol-Gel Sci. Technol. 8 (1997) 353.

[2] M.O. Prado, E.D. Zanotto, R. Müller, J. Non-Cryst. Solids 279 (2001) 169.

[3] E.D. Zanotto, M.O. Prado, Phys. Chem. Glasses 42 (2001) 191.

[4] M.O. Prado, C. Fredericci, E.D. Zanotto, J. Non-Cryst. Solids 331 (2003) 145

[5] M.O. Prado, E.D. Zanotto, C. Fredericci, J. Mater. Res. 18 (2003) 1347.

[6] M.O. Prado, C. Fredericci, E.D. Zanotto, J. Non-Cryst. Solids 331 (2003) 157.

[7] R. Müller, Glastech. Ber. Glass Sci. Technol. 67C (1994) 93.

[8] M.O. Prado, C. Fredericci, E.D. Zanotto, Phys. Chem. Glasses 43 (2002) 215

[9] M.L.F. Nascimento, E.D. Zanotto, Phys. Rev. B 73 (2006) 024209.

[10] M.L.F. Nascimento, E.D. Zanotto, Phys. Chem. Glasses: Eur. J. Glass Sci. Technol. B 48 (2007) 201.

[11] M.L.F. Nascimento, E.B. Ferreira, E.D. Zanotto, J. Chem. Phys. 121 (2004) 8924.

[12] J.J. Frenkel, J. Phys. (USSR) 9 (1945) 385.

[13] J.K. Mackenzie, R. Shuttleworth, Proc. Phys. Soc. Lond., Sect. B, 62 (1949) 833.

[14] E.A. Giess, J.P. Fletcher, L.W. Herron, J. Am. Ceram. Soc. 67 (1984) 549.

[15] G.W. Scherer, J. Am. Ceram. Soc. 60 (1977) 236.

[16] M.L.F. Nascimento, PhD thesis, Current problems on crystal nucleation and growth, and viscous flow in glasses, Federal University of São Carlos, 2004 (in Portuguese).

[17] M.L.F. Nascimento, C. Aparicio, J. Phys. Chem. Solids 68 (2007) 104

[18] M.L.F. Nascimento, C. Aparicio, Phys. B, Condens. Matter 398 (2007) 71.

[19] S. Reinsch, R. Müller, M.L.F. Nascimento, E.D. Zanotto, Proceedings of XX International Congress on Glass, Japan, 2004.

[20] C. Casado, MSc dissertation, Federal University of São Carlos, 2003.

[21] C. Paucar, personal communication, 2004.

[22] M.J. Pascual, A. Durán, M.O. Prado, E.D. Zanotto, J. Am. Ceram. Soc. 88 (2005) 1427.

[23] R. Müller, E.D. Zanotto, V.M. Fokin, J. Non-Cryst. Solids 274 (2000) 208.

[24] SciGlass 6.5, SciGlass Dictionary, 2000-2004, Scivision. 